\title{
Fault Diagnosis of Nonlinear Analog Circuit Based on Generalized Frequency Response Function and LSSVM Classifier Fusion
}

\author{
Jialiang Zhang (D) \\ School of Electronic Information Engineering, Xi'an Technological University, Xi'an 710021, China \\ Correspondence should be addressed to Jialiang Zhang; zj1512@163.com
}

Received 23 June 2020; Revised 3 September 2020; Accepted 13 September 2020; Published 22 September 2020

Academic Editor: Frederic Kratz

Copyright (c) 2020 Jialiang Zhang. This is an open access article distributed under the Creative Commons Attribution License, which permits unrestricted use, distribution, and reproduction in any medium, provided the original work is properly cited.

\begin{abstract}
For fault diagnosis of nonlinear analog circuit, a novel method based on generalized frequency response function (GFRF) and least square support vector machine (LSSVM) classifier fusion is presented. The sinusoidal signal is used as the input of analog circuit, and then, the generalized frequency response functions are estimated directly by the time-domain formulations. The discrete Fourier transform of measurement data is avoided. After obtaining the generalized frequency response functions, the amplitudes of the GFRFs are chosen as the fault feature parameters. A classifier fusion algorithm based on least square support vector machine (LSSVM) is used for fault identification. Two LSSVM multifault classifiers with different kernel functions are constructed as subclassifiers. Fault diagnosis experiments of resistor-capacitance (RC) circuit and Sallen Key filter are carried out, respectively. The results show that the estimated GFRFs of the circuit are accurate, and the fault diagnosis method can get high recognition rate.
\end{abstract}

\section{Introduction}

Analog circuits are widely used in various electronic systems and play a very important role. When the fault occurred, they will affect the performance of the relevant electronic systems and even make the systems completely invalid. Therefore, it is of great significance to research the fault diagnosis of analog circuits. In order to study the problem of fault diagnosis of analog circuits, a variety of diagnosis methods have been proposed [1-3]. A wavelet neural network diagnosis model is presented to improve the diagnosis capability of soft fault for analog circuit, and the adaptive unscented Kalman filter is used to optimize the parameters of wavelet neural network [4]. The fuzzy classifier is constructed to diagnose the faults of analog electronic circuit, and the simulation before test approach is used to obtain signature parameters by analyzing the frequency response [5]. By using kurtosis and entropy as feature information, the diagnosis of analog circuit is studied based on artificial neural network [6]. A modular fault diagnosis system for analog electronic circuits is designed based on wavelet transform and neural network [7]. The features are obtained by wavelet fractal analysis and kernel principal component analysis, and novel linear ridgelet network approach is used for analog circuit fault diagnosis [8]. The relationship between the input and output of analog circuit is very complex, which is often nonlinear. There are still many problems in fault diagnosis of nonlinear analog circuits, especially in feature information acquisition and fault identification.

Volterra series is an important mathematical tool for theoretical analysis and practical application of nonlinear systems. Volterra frequency-domain kernel, also known as generalized frequency response function, is an extension of frequency response function of linear system in nonlinear system. It provides a description method of frequencydomain characteristics of nonlinear system, which can reflect the inherent characteristics of the system [9-14]. The generalized random signal is used as the input, and the first two-order GFRFs of the nonlinear system are solved according to the input spectrum and the output spectrum [15]. The measurement of Volterra frequency-domain kernel for nonlinear dynamic $3 \times 3$ multi-input multi-output system is studied, and the characteristic of Volterra frequency-domain kernel is analyzed [16]. In [17], a new complex orthogonal least square algorithm is used to truncate the Volterra series and estimate the generalized 
frequency response function. A novel method for highorder frequency response function measurement of nonlinear system is presented based on cross-correlation technology, and the system input is sinusoidal signal [18]. For the prediction of nonlinear aeroelastic vibration response and flutter, a frequency component identification method based on correlation analysis is used to estimate the GFRFs [19]. In [20], a two-step strategy is used to estimate the generalized frequency response function of the nonlinear controlled plant in the closed-loop state. For the above estimation methods, the input and output data need to be Fourier-transformed in the process of solving GFRFs. An estimation method based on time-domain data is introduced to calculate GFRFs of single-input single-output nonlinear systems [21]. It does not need Fourier transform for time-domain data and reduces the computational complexity. The feature information in frequency domain is obtained by the generalized frequency response function, which can be used for fault diagnosis of nonlinear system. In [22], a new fault diagnosis method for nonlinear analog circuit based on simplified estimation of GFRFs and hybrid-kernel least squares support vector machine is proposed.

After obtaining the frequency-domain characteristic information of nonlinear system by using the generalized frequency response function, it is very important to identify the fault accurately according to the data. As an effective machine learning method, support vector machine can be used to study classification problems [23, 24]. It can realize small sample learning and has good generalization ability. It has been used in fault diagnosis of many systems [25-28]. In [29], for the real-time fault diagnosis of high-voltage circuit breakers, fault detection is carried out by adaptive KPCA, and then fault recognition is completed by SVM classifier. The mechanical fault diagnosis of high voltage circuit breaker is studied by combining genetic algorithm with support vector machine [30]. The wavelet theory is used to obtain feature information, and then the support vector machine is used to detect bearing fault of asynchronous motor [31]. The state of asynchronous motor is monitored based on Hilbert-Park transform and support vector machine [32]. By combining support vector machine and multisensor data fusion method, the fault diagnosis of the motor is studied [33]. In [34], the statistical features are classified by support vector machine, and the fault diagnosis of the hydraulic brake system is carried out. In [35], a modified support vector machine classifier is used to analyze the fault of high voltage circuit breaker based on the waveform of coil current and travel waveforms. A twostage scheme based on support vector machine is presented for the fault detection and classification of electrical pitch drives in offshore wind farms [36]. The least squares support vector machine is proposed by changing the risk function of support vector machine [37]. The training of LSSVM only needs to solve a system of linear equations, which reduces the computational complexity.

In this paper, a new fault diagnosis method of nonlinear analog circuit is proposed based on generalized frequency response function and LSSVM classifier fusion algorithm. In order to simplify calculation, the sinusoidal signal is used as the input, and the time-domain measurements are used to directly estimate the GFRFs. After obtaining the generalized frequency response functions, their amplitudes of GFRFs are chosen as the feature parameters of analog circuits. Two multifault classifiers are constructed as subclassifier using least square support vector machine, where the kernel functions are polynomial function and Gaussian radial basis function, respectively. By using a classifier fusion algorithm based on the binary LSSVM classification function of the subclassifiers, the diagnosis result can be derived. Two experiments for the fault diagnosis of RC circuit and Sallen Key filter verified the effectiveness of the proposed method.

\section{Simplified Estimation of GFRFs Using Time- Domain Measurements}

The Volterra series is an extension of Taylor series and is widely used to describe and analyze nonlinear systems. A single-input single-output nonlinear system can be expressed as a Volterra series:

$$
\begin{aligned}
y(t)= & \sum_{n=1}^{\infty} y_{n}(t) \\
y_{n}(t)= & \int_{-\infty}^{\infty} \int_{-\infty}^{\infty} \cdots \int_{-\infty}^{\infty} h_{n}\left(\tau_{1}, \tau_{2}, \ldots, \tau_{n}\right) \\
& \cdot \prod_{i=1}^{n} u\left(t-\tau_{i}\right) d \tau_{1}, \ldots, d \tau_{n}
\end{aligned}
$$

where $u(t) \in R$ is the input, $y(t) \in R$ is the output, and $h_{n}\left(\tau_{1}, \tau_{2}, \ldots, \tau_{n}\right)$ is the $n$th order Volterra kernel.

The multidimensional Fourier transform of the nth order Volterra kernel $h_{n}\left(\tau_{1}, \tau_{2}, \ldots, \tau_{n}\right)$ is given by

$$
\begin{aligned}
& H_{n}\left(j \omega_{1}, j \omega_{2}, \ldots, j \omega_{n}\right)=\int_{-\infty}^{\infty} \int_{-\infty}^{\infty} \ldots \int_{-\infty}^{\infty} \\
& h_{n}\left(\tau_{1}, \tau_{2}, \ldots, \tau_{n}\right) e^{-j\left(\omega_{1} \tau_{1}+\omega_{2} \tau_{2}+\cdots+\omega_{n} \tau_{n}\right)} d \tau_{1}, \ldots, d \tau_{n},
\end{aligned}
$$

where $H_{n}\left(j \omega_{1}, j \omega_{2}, \ldots, j \omega_{n}\right)$ represents the $n$ th-order Volterra frequency-domain kernel, also known as the $n$ thorder generalized frequency response function, which is a mapping form of the $n$ th-order Volterra kernel in the frequency domain.

The frequency-domain output of a single-input singleoutput nonlinear system can be expressed as a generalized frequency response function:

$$
\begin{aligned}
Y(j \omega)= & \sum_{n=1}^{\infty} Y_{n}(j \omega), \\
Y_{n}(j \omega)= & \frac{1}{(2 \pi)^{n-1}} \int_{-\infty}^{\infty} \cdots \int_{-\infty}^{\infty} H_{n}\left(j \omega-j \omega_{1}-\cdots-j \omega_{n-1},\right. \\
& \left.\cdot j \omega_{1}, \ldots, j \omega_{n-1}\right) \cdot U\left(j \omega-j \omega_{1}-\cdots-j \omega_{n-1}\right) \\
& \cdot U\left(j \omega_{1}\right), \ldots, U\left(j \omega_{n-1}\right) d \omega_{1}, \ldots, d \omega_{n-1},
\end{aligned}
$$


where $Y(j \omega)$ is the frequency-domain output of the system, $Y_{n}(j \omega)$ is the $n$th order frequency-domain output of the system, and $U(\cdot)$ is the input spectrum.

The discrete form of the single-input single-output nonlinear system described by equations (1) and (2) can be expressed as

$$
\begin{aligned}
y(k) & =\sum_{n=1}^{\infty} y_{n}(k), \\
y_{n}(k) & =\sum_{n=1}^{\infty} \sum_{-\infty}^{\infty} \cdots \sum_{-\infty}^{\infty} h_{n}\left(\tau_{1}, \tau_{2}, \cdots, \tau_{n}\right) \prod_{i=1}^{n} u\left(t-\tau_{i}\right), \quad k \in Z .
\end{aligned}
$$

If a weakly nonlinear system can be described by the first-order Volterra series, the frequency-domain form of the discrete Volterra model of the system is given by

$$
Y(\omega)=H_{1}(\omega) U(\omega)+\sum_{p+q=\omega} H_{2}(p, q) U(p) U(q),
$$

where $H_{1}(\omega)$ is the first-order generalized frequency response function, $\mathrm{H}_{2}(p, q)$ is the second order generalized frequency response function, and $U(\cdot)$ is the input spectrum.

Let the input be a sinusoidal signal $u(t)=A \cos \omega t$, and then, the output of the nonlinear system can be expressed as [21]

$$
\begin{aligned}
y(k)= & A \operatorname{Re}\left\{H_{1}(\omega) e^{j \omega k}\right\}+2\left(\frac{A}{2}\right)^{2} \operatorname{Re}\left\{H_{2}(\omega, \omega) e^{j 2 \omega k}\right\} \\
& +2\left(\frac{A}{2}\right)^{2} \operatorname{Re}\left\{H_{2}(\omega,-\omega)\right\}+e(k),
\end{aligned}
$$

where $\operatorname{Re}(\cdot)$ is the real part of a complex number, and $e(k)$ is the zero mean Gaussian white noise.

Define

$$
\begin{aligned}
H_{1}(\omega) & =R_{1}(\omega)+j I_{1}(\omega), \\
H_{2}(\omega, \omega) & =R_{2}(\omega, \omega)+j I_{2}(\omega, \omega), \\
H_{2}(\omega,-\omega) & =R_{0},
\end{aligned}
$$

where $R_{1}(\omega)$ and $I_{1}(\omega)$ represent the real part and imaginary part of $H_{1}(\omega)$, respectively, $R_{2}(\omega, \omega)$ and $I_{2}(\omega, \omega)$ represent the real part and imaginary part of $H_{2}(\omega, \omega)$, respectively, and $R_{0}$ is a constant.

From the equations (7) (10), the following can be obtained:

$$
\begin{aligned}
y(k)= & A R_{1}(\omega) \cos (k \omega)+A I_{1}(\omega)[-\sin (k \omega)] \\
& +2\left(\frac{A}{2}\right)^{2} R_{2}(\omega, \omega) \cos (2 k \omega)-2\left(\frac{A}{2}\right)^{2} \\
& \cdot I_{2}(\omega, \omega) \sin (2 k \omega)+2\left(\frac{A}{2}\right)^{2} R_{0}+e(k) .
\end{aligned}
$$

For $k=1,2, \ldots, N$, equation (11) can be written as the matrix form:

$$
\mathbf{Y}=\mathbf{X} \boldsymbol{\theta}+\mathbf{E},
$$

where $\mathbf{Y}=[y(1), y(2), \ldots, y(N)]^{T}, \theta=\left[R^{\prime}, I^{\prime}, R_{2}, I_{2}, R_{0}\right]^{T}$, $\mathbf{E}=[e(1), e(2), \ldots, e(N)]^{T}$, and

$$
X=\left[\begin{array}{ccccc}
A \cos (\omega) & -A \sin (\omega) & 2\left(\frac{A}{2}\right)^{2} \cos (2 \omega) & -2\left(\frac{A}{2}\right)^{2} \sin (2 \omega) & 2\left(\frac{A}{2}\right)^{2} \\
A \cos (2 \omega) & -A \sin (2 \omega) & 2\left(\frac{A}{2}\right)^{2} \cos (4 \omega) & -2\left(\frac{A}{2}\right)^{2} \sin (4 \omega) & 2\left(\frac{A}{2}\right)^{2} \\
\vdots & & \\
A \cos (N \omega) & -A \sin (N \omega) & 2\left(\frac{A}{2}\right)^{2} \cos (2 N \omega) & -2\left(\frac{A}{2}\right)^{2} \sin (2 N \omega) & 2\left(\frac{A}{2}\right)^{2}
\end{array}\right] .
$$

Equation (12) can be solved by using standard least squares principle:

$$
\widehat{\theta}=\left(\mathbf{X}^{T} \mathbf{X}\right)^{-1} \mathbf{X}^{T} \mathbf{Y}
$$

where $\widehat{\theta}=\left[\widehat{R}_{1}, \widehat{I}_{1}, \widehat{R}_{2}, \widehat{I}_{2}, \widehat{R}_{0}\right]^{T}$.

The generalized frequency response function can reflect the essential characteristics of nonlinear systems. For a nonlinear system, the GFRF is different in different fault modes. Therefore, fault diagnosis of nonlinear analog circuits can be realized by using GFRF to obtain characteristic information. After estimating the generalized frequency response functions of the nonlinear analog circuit by equation (14), their amplitudes are chosen as the feature parameters for fault diagnosis in this paper.

\section{Multifault Classifier Design Based on LSSVM Classifier Fusion}

Support vector machine is a machine learning method developed on the basis of statistical learning theory. It uses the principle of structural risk minimization to improve the 
generalization ability, which can solve the problems of small samples and local minima and can effectively classify data. However, the support vector machine needs to solve the quadratic programming problem so that the calculation is very complicated. Least squares support vector machine is a deformed support vector machine algorithm. The training of LSSVM only requires solving a system of linear equations, which is easy to solve and has high training efficiency.

Let the training sample data be $S_{1}:\left\{\left(\mathbf{x}_{i}, y_{i}\right)\right\}_{i=1}^{M}$, where $\mathbf{x}_{i} \in \mathbf{R}^{p}$ is the input space sample, $y_{i} \in\{-1,1\}$ is the class label, and $M$ is the sample size. The binary classification problem of least squares support vector machine can be described as

$$
\begin{aligned}
& \min \frac{1}{2} \boldsymbol{\omega}^{\mathrm{T}} \boldsymbol{\omega}+\frac{1}{2} C \sum_{i=1}^{M} \xi_{i}^{2} \\
& \text { s.t. } y_{i}\left[\boldsymbol{\omega}^{\mathrm{T}} \varphi\left(\mathbf{x}_{i}\right)+b\right]=1-\xi_{i}, \quad i=1,2, \ldots, M,
\end{aligned}
$$

where $\omega$ is the weight vector of the classification hyperplane, $C>0$ is the penalty factor, $\xi_{i}$ is the relaxation variable, $\varphi(\cdot)$ is the nonlinear mapping, and $b$ is the classification threshold.

Define the Lagrange function as

$$
\begin{aligned}
L\left(\boldsymbol{\omega}, b, \xi_{i}, \alpha_{i}\right)= & \frac{1}{2} \boldsymbol{\omega}^{\mathrm{T}} \boldsymbol{\omega}+\frac{1}{2} C \sum_{i=1}^{M} \xi_{i}^{2} \\
& -\sum_{i=1}^{M} \alpha_{i}\left\{y_{i}\left[\boldsymbol{\omega}^{\mathrm{T}} \varphi\left(\mathbf{x}_{i}\right)+b\right]-1+\xi_{i}\right\},
\end{aligned}
$$

where $\alpha_{i} \geq 0$ is the Lagrange multiplier.

Letting the partial derivatives of the Lagrange function for $\omega, b, \xi_{i}$ and $\alpha_{i}$ be zero, the constraint optimization problem of LSSVM can be transformed into a system of linear equations:

$$
\left[\begin{array}{ll}
0 & \mathbf{Y}^{T} \\
\mathbf{Y} & \mathbf{H}
\end{array}\right] \cdot\left[\begin{array}{l}
b \\
\boldsymbol{\alpha}
\end{array}\right]=\left[\begin{array}{l}
0 \\
\mathbf{1}
\end{array}\right],
$$

where $\mathbf{Y}=\left[y_{1}, y_{2}, \cdots, y_{M}\right]^{T}, \mathbf{H}=\Omega+c^{-1} \cdot \mathbf{I}, \Omega$ is the $M$ dimensional symmetric matrix, $\Omega_{i, j}=y_{i} y_{j} K\left(\mathbf{x}_{i}, \mathbf{x}_{j}\right), K(\cdot, \cdot)$ is the kernel function, $\mathbf{I}$ is the $M$-dimensional unit matrix, $\alpha=\left[\alpha_{1}, \alpha_{2}, \cdots, \alpha_{M}\right]^{T}$ is the Lagrange multiplier vector, and $\mathbf{1}=[1,1, \ldots, 1]^{T}$.

The decision function is given by

$$
y(\mathbf{x})=\operatorname{sign}\left[\sum_{i=1}^{M} \alpha_{i} y_{i} K\left(\mathbf{x}, \mathbf{x}_{i}\right)+b\right],
$$

where $\mathbf{x}$ is the sample vector to be classified.

For the classification of nonlinear data, the SVM classifier maps the feature data to a high-dimensional feature space using a nonlinear function and then completes the classification. Therefore, when using the least squares support vector machine to construct a classifier, the choice of kernel function is very important and will directly affect the classification result. Commonly used kernel functions include polynomial kernel function and Gaussian radial basis kernel function. When traditional LSSVM deals with the multiclassification problem, one type of function is selected as the kernel function, and one classifier is constructed for classification. Because different kernel functions have different characteristics, their classification performance for the same feature data is also different.

In order to improve the fault recognition rate, a LSSVM classifier fusion method is adopted to identify faults in nonlinear analog circuits. Polynomial kernel function and Gaussian radial basis kernel function are used to construct two LSSVM classifiers as subclassifiers. Then, the two subclassifiers are fused to obtain the diagnosis result. The polynomial kernel function is a global kernel function, and the Gaussian radial basis kernel function is a local kernel function. Therefore, this LSSVM fusion algorithm can take into account the global characteristics and local characteristics of the feature data obtained through the generalized frequency response functions of the nonlinear analog circuits.

The structures of the designed LSSVM subclassifiers are "one-against-one," and the voting method is used for fault decisions of subclassifiers. The structure of nonlinear circuit fault diagnosis based on LSSVM fusion is shown in Figure 1, where $N$ is the number of categories of data to be classified.

The fusion strategy of support vector machine has a great impact on the accuracy of the classifier. Therefore, choosing an appropriate fusion algorithm is very important for fault identification.

According to decision function (18) of the binary SVM, define

$$
y^{\prime}(\mathbf{x})=\alpha_{i} y_{i} K\left(\mathbf{x}, \mathbf{x}_{i}\right)+b
$$

The function $y^{\prime}(\mathbf{x})$ represents the distance from sample $\mathbf{x}$ to the optimal hyperplane, which can indirectly reflect the degree of support for decision results. In this paper, the weight coefficients are obtained on the basis of the function $y^{\prime}(\mathbf{x})$ of each binary SVM in the subclassifier, and then the weighted fusion is performed to obtain the fault identification result.

Assume that the fault modes of the nonlinear analog circuit are $f_{1}, f_{2}, \ldots, f_{N}, N$ is the number of modes, the obtained GFRFs amplitude data of the nonlinear analog circuit is $\mathbf{x}$, and the decision results of the two LSSVM subclassifiers are $D_{1}$ and $D_{2}$. Define the weight of the LSSVM subclassifier as

$$
w_{j}=\sum_{k=1}^{V_{j}}\left|\sum_{i=1}^{M} \alpha_{j k i} y_{j k i} K_{j k}\left(\mathbf{x}, \mathbf{x}_{j k i}\right)+b_{j k}\right|,
$$

where $j=1,2, M$ is the training sample capacity for each binary LSSVM classifier in subclassifier $j, V_{j}$ is the number of binary LSSVM classifiers with a decision result of $D_{j}, \alpha_{j k i}$ and $y_{j k i}$ are the Lagrangian multiplier and class label of the $k$ th binary LSSVM classifier in subclassifier $j$, and $K_{j k}\left(\mathbf{x}, \mathbf{x}_{j k i}\right)$ and $b_{j k}$ are the kernel function and the classification threshold of the $K$-th binary LSSVM classifier in the subclassifier $j$.

By fusing the decision results of the two subclassifiers, the fault identification result is given by 


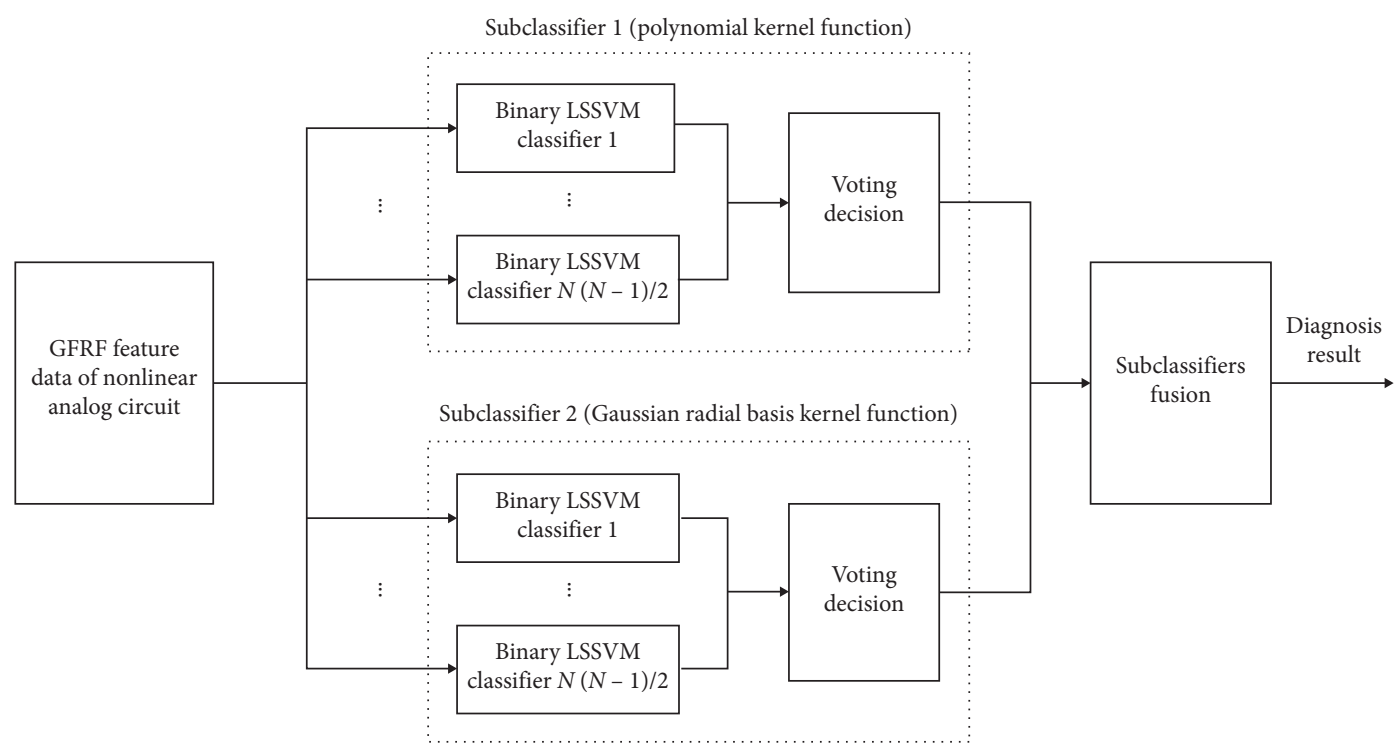

FIgURE 1: The structure of nonlinear circuit fault diagnosis based on LSSVM fusion.

$$
\begin{gathered}
D=\underset{f_{l} \in Q}{\arg \max }\left(\sum_{j=1}^{2} w_{j} \delta_{j, l}\right), \\
\delta_{j, l}= \begin{cases}1, & D_{j}=f_{l}, \\
0, & D_{j} \neq f_{l},\end{cases}
\end{gathered}
$$

where $Q \subseteq\left\{f_{1}, f_{2}, \ldots, f_{N}\right\}$ is the set of fault modes formed by the decision results of the two LSSVM subclassifiers.

The result of subclassifier is given by

$$
\begin{gathered}
D_{j}=\underset{f_{k}}{\arg \max } \sum_{m=1}^{p} A_{j k m}, \quad(k=1,2, \ldots, N), \\
A_{j k m}=\left\{\begin{array}{ll}
1 & D_{j m}=f_{k} \\
0 & D_{j m} \neq f_{k}
\end{array}, \quad(m=1,2, \ldots, p),\right.
\end{gathered}
$$

where $D_{j m}$ is the decision result of the $m$ th binary LSSVM classifier of subclassifier $j$, and $p=N((N-1) / 2)$.

After obtaining the frequency-domain feature information of the nonlinear analog circuit based on the generalized frequency response functions, the fusion algorithm of least square support vector machine is used for fault diagnosis. The fault diagnosis steps are as follows:

(i) Step 1. Input the frequency-domain feature vector $\mathbf{x}$ of the nonlinear analog circuit into subclassifier 1 and subclassifier 2 , respectively, and solve the value of the $\alpha_{j k i} y_{j k i} K_{j k}\left(\mathbf{x}, \mathbf{x}_{j k i}\right)+b_{j k},(k=1, \ldots, N((N-$ 1)/2)) of the binary LSSVM classifier in the subclassifiers

(ii) Step 2. For each LSSVM subclassifier, calculate the decision result by equation (18), and then make a voting based on all of the results

(iii) Step 3. According to the classification function of the binary LSSVMs in the subclassifiers, calculate the weights of the LSSVM subclassifiers by using equation (20)

(iv) Step 4. According to the decision results $D_{1}$ and $D_{2}$ of the subclassifier, and the corresponding weights $w_{1}$ and $w_{2}$, calculate the fusion result by equations (21) (24)

Because the fault diagnosis method of nonlinear analog circuits based on LSSVM fusion uses the classification function of binary LSSVM to obtain the weight of the subclassifier, the classification reliability of the two subclassifiers with different types of kernel functions is fully considered.

\section{Experiment and Analysis}

Two experiments of nonlinear analog circuits are used to verify the effectiveness of the diagnosis method.

4.1. Resistor-Capacitance (RC) Circuit. A resistor-capacitance circuit is shown in Figure 2, where $u_{s}$ is the supply voltage, $R_{1}$ and $R_{2}$ are the resistances, and $C$ is the capacitance. The relationship between voltage and current of the nonlinear resistance $R_{2}$ is $i_{2}=a_{1} u+a_{2} u^{2}$, where $a_{1}=1 \times 10^{-3}$ and $a_{2}=5 \times 10^{-4}$

According to Kirchhoff's voltage law and current law, we can obtain

$$
\left\{\begin{array}{l}
u_{\mathrm{in}}(t)=R_{1} i_{1}(t)+u(t), \\
i_{1}(t)=i_{2}(t)+i_{3}(t)
\end{array}\right.
$$

where $i_{3}(t)=C(\mathrm{~d} u(t) / \mathrm{d} t)$.

Let the power supply voltage $u_{\text {in }}(t)$ be the input and let the voltage $u(t)$ on $R_{2}$ be the output. The nonlinear differential equation can be obtained as

$$
\frac{\mathrm{d} u(t)}{\mathrm{d} t}+b_{1} u(t)+b_{2} u^{2}(t)=b_{3} u_{\text {in }}(t),
$$




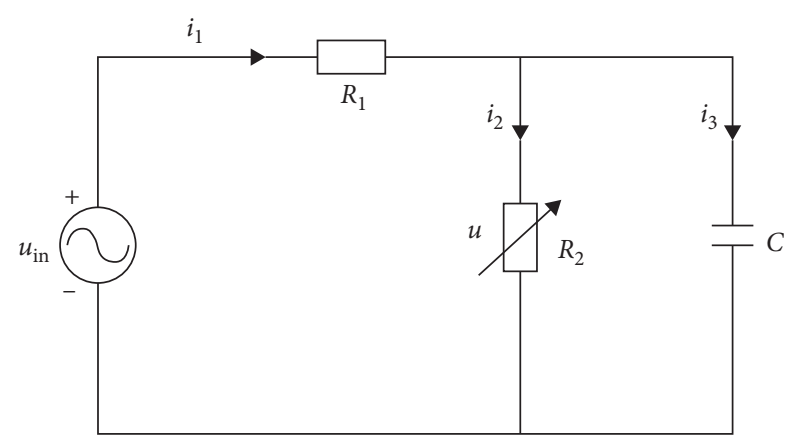

FIgURE 2: A resistor-capacitance circuit.

where $\quad b_{1}=\left(\left(a_{1} R_{1}+1\right) / R_{1} C\right), \quad b_{2}=\left(a_{2} / C\right), \quad$ and $b_{3}=\left(1 / R_{1} C\right)$.

The nominal values of components in the analog circuit system are $C=10^{-4} \mathrm{~F}$ and $R_{1}=10^{3} \Omega$. The simulation experiment has been performed using MATLAB R2014a. The CPU clock speed of the computer is $2.3 \mathrm{GHz}$, and the main memory is $8 \mathrm{~GB}$.

Let the input signal be $u_{\text {in }}(t)=\cos 2 \pi t$. The sampling frequency is $100 \mathrm{~Hz}$, and sampling length is $10 \mathrm{~s}$. The output spectrum of the nonlinear circuit is shown in Figure 3. There are significant peaks at frequencies $1 \mathrm{~Hz}$ and $2 \mathrm{~Hz}$, which are 0.48 and 0.03 , respectively. The output of analog circuit has obvious second harmonics so that the circuit has obvious nonlinear characteristics. Because the harmonic amplitudes above the third harmonic are approximately equal to 0 , the analog circuit can be described by the first two-order generalized frequency response functions.

For the nonlinear circuit system described by Equation (26), the actual value of GFRFs can be derived [38]:

$$
\begin{gathered}
H_{1}(j \omega)=\frac{b_{3}}{j \omega+b_{1}}, \\
H_{2}\left(j \omega_{1}, j \omega_{2}\right)=\frac{-b_{2} H_{1}\left(j \omega_{1}\right) H_{1}\left(j \omega_{2}\right)}{j\left(\omega_{1}+\omega_{2}\right)+b_{1}} .
\end{gathered}
$$

When the output noise is Gaussian white noise with signal-to-noise ratios (SNR) of $35 \mathrm{~dB}, 30 \mathrm{~dB}$, and $25 \mathrm{~dB}$, respectively, the simplified estimation method is used to obtain the generalized frequency response functions of the analog circuit. The output of the nonlinear circuit is shown in Figure 4. As the SNR gradually decreases, the deformation of the output becomes more and more obvious. The estimation results are shown in Table 1 , where $\omega=2 \pi$. Under different signal-to-noise ratios, the GFRFs of nonlinear analog circuit can be accurately estimated. As the signal-to-noise ratio decreases, the estimation error gradually increases.

Assume that, under normal conditions, the variation ranges of $C$ and $R_{1}$ are within $5 \%$. If the actual value of $C$ or $R_{1}$ differs from the nominal value by $50 \%$, the component will fail. The analog circuit contains five fault modes, and the fault description is shown in Table 2.

The Monte Carlo method is used for fault diagnosis simulation experiment of the analog circuit. The output

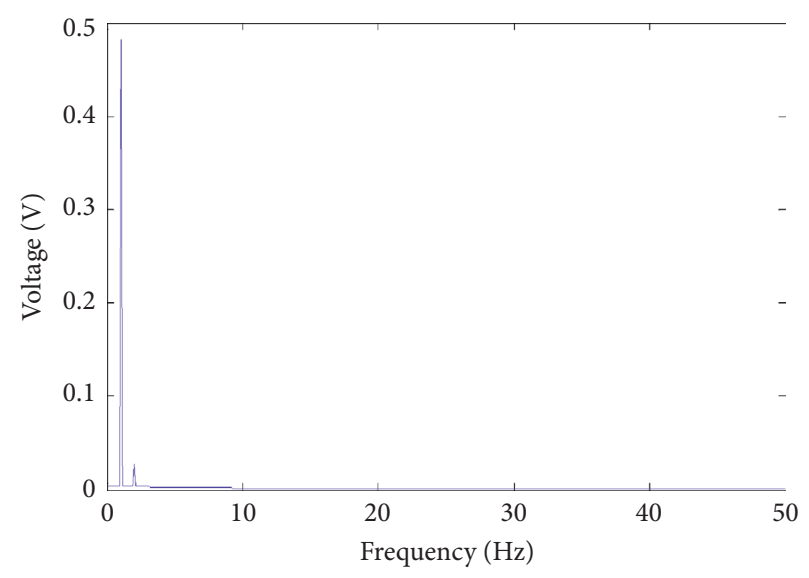

Figure 3: The output spectrum of analog circuit.

noise is Gaussian white noise, and the SNR is $25 \mathrm{~dB} .200$ sets of input and output sample data of the analog circuit are collected for each fault. The amplitudes of the GFRFs $H_{1}(\omega)$, $H_{2}(\omega, \omega)$ and $H_{2}(\omega,-\omega)$ are solved by the proposed simplified estimation method. The amplitude distributions of the five fault types are shown in Figure 5.

The 100 sets of sample data are used as training samples, and the remaining 100 sets are used as test samples. The fault diagnosis of the analog circuit is carried out by the LSSVM fusion algorithm and the traditional single LSSVM multiclassification algorithm, respectively. The order of the polynomial function is 2 , and the width of the Gaussian radial basis function is $4 \times 10^{-3}$. The fault diagnosis steps of the LSSVM fusion algorithm are shown in Section 3, and the results are obtained using Step 1 to Step 4.

The fault recognition rate is shown in Table 3 . The recognition rates of the LSSVM fusion algorithm are higher than those of the traditional single LSSVM classifiers.

4.2. Sallen Key Filter. A Sallen Key filter circuit is shown in Figure 6, where $R_{1}=1 \mathrm{k} \Omega, R_{2}=3 \mathrm{k} \Omega, R_{3}=2 \mathrm{k} \Omega$, $R_{4}=4 \mathrm{k} \Omega \mathrm{m}, R_{5}=4 \mathrm{k} \Omega, C_{1}=5 \mathrm{nF}, C_{2}=5 \mathrm{nF}$, and the input voltage is $u=\cos 2000 \pi t \mathrm{~V}$.

Assuming that the actual value of $R_{4}$ or $R_{5}$ differs from the nominal value by $50 \%$, the component will fail. There are five fault modes, normal (F0), R4 increased by $50 \%$ (F1), R4 decreased by $50 \%$ (F2), R5 increased by $50 \%$ (F3), and R5 decreased by $50 \%$ (F4). The Monte Carlo method is used for fault diagnosis experiment of the Sallen Key filter circuit, and the tolerances of the circuit components are $5 \%$. The input and output data of the circuit are obtained using Multisim 10. The diagnosis algorithm has been performed using MATLAB R2014a.

The output noise is Gaussian white noise, and the SNR is $40 \mathrm{~dB}$. The sampling frequency is $105 \mathrm{~Hz}$, and sampling length is $10 \mathrm{~ms}$. The output of the Sallen Key filter in normal state is shown in Figure 7.

200 sets of input and output sample data of the analog circuit are collected for each fault. The amplitudes of the GFRFs $H_{1}(\omega), H_{2}(\omega, \omega)$ and $H_{2}(\omega,-\omega)$ are solved by the proposed simplified estimation method, where $\omega=2000 \pi$. 


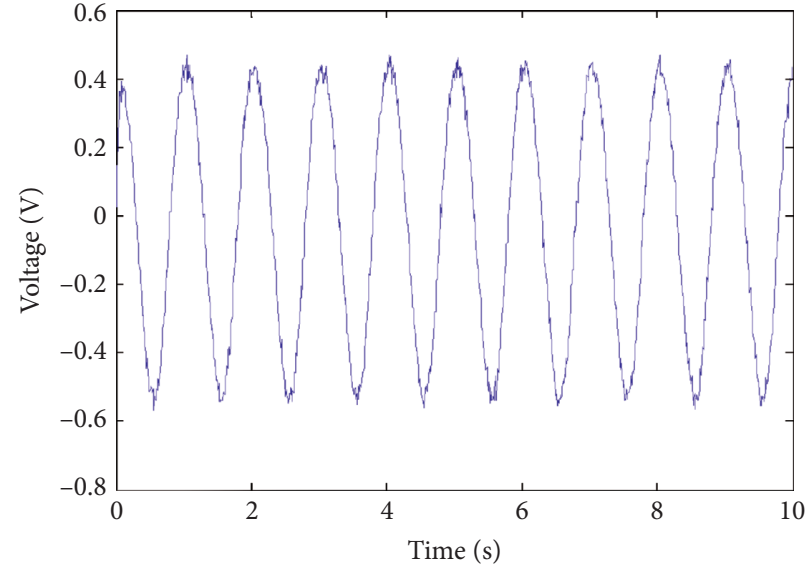

(a)

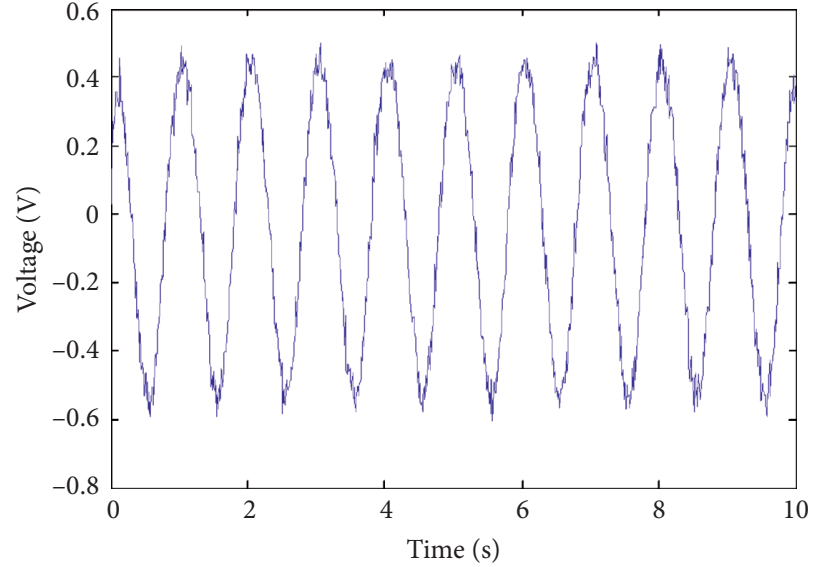

(b)

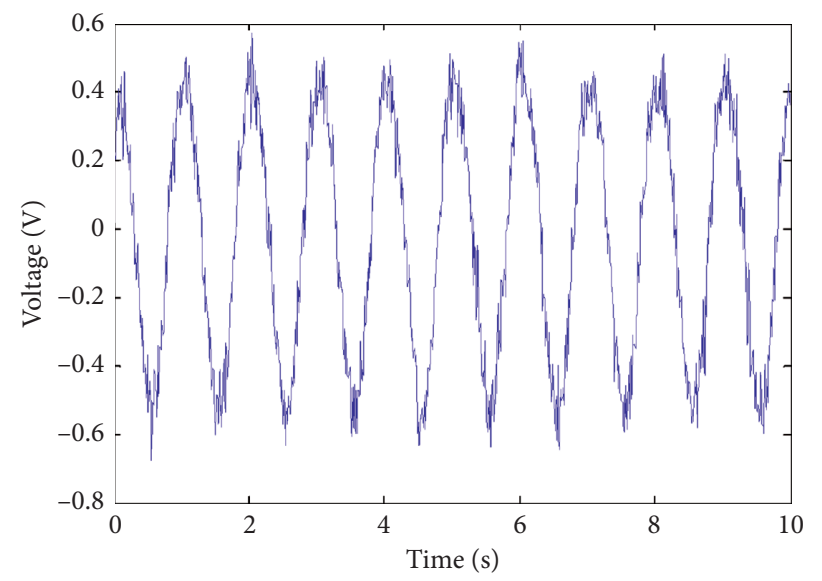

(c)

Figure 4: The output of analog circuit: (a) $\mathrm{SNR}=35 \mathrm{~dB}$, (b) $\mathrm{SNR}=30 \mathrm{~dB}$, (c) $\mathrm{SNR}=25 \mathrm{~dB}$.

TABLE 1: The estimation results of GFRFs.

\begin{tabular}{lcccc}
\hline GFRFs & Actual value [38] & \multicolumn{3}{c}{ Estimated value } \\
& & SNR $=35 \mathrm{~dB}$ & SNR $=30 \mathrm{~dB}$ & $\mathrm{SNR}=25 \mathrm{~dB}$ \\
\hline$H_{1}(\omega)$ & $0.455-0.143 i$ & $0.462-0.150 i$ & $0.459-0.152 i$ & $0.459-0.153 i$ \\
$H_{2}(\omega, \omega)$ & $-0.019+0.044 i$ & $-0.019+0.048 i$ & $-0.020+0.048 i$ & $-0.023+0.050 i$ \\
$H_{2}(\omega,-\omega)$ & -0.057 & -0.059 & -0.060 & -0.060 \\
\hline
\end{tabular}

TABLE 2: The fault description of analog circuit.

\begin{tabular}{lcc}
\hline Fault number & Fault type & Parameter value \\
\hline F0 & Normal & $C \in\left[0.95 \times 10^{-4} F \sim 1.05 \times 10^{-4} F\right], R_{1} \in\left[0.95 \times 10^{3} \Omega \sim 1.05 \times 10^{3} \Omega\right]$ \\
F1 & $C$ increased & $C \in\left[1.45 \times 10^{-4} F \sim 1.55 \times 10^{-4} F\right], R_{1} \in\left[0.95 \times 10^{3} \Omega \sim 1.05 \times 10^{3} \Omega\right]$ \\
F2 & $C$ decreased & $C \in\left[0.45 \times 10^{-4} F \sim 0.55 \times 10^{-4} F\right], R_{1} \in\left[0.95 \times 10^{3} \Omega \sim 1.05 \times 10^{3} \Omega\right]$ \\
F3 & $C \in\left[0.95 \times 10^{-4} F \sim 1.05 \times 10^{-4} F\right], R_{1} \in\left[1.45 \times 10^{3} \Omega \sim 1.55 \times 10^{3} \Omega\right]$ \\
F4 & $R_{1}$ increased & $C \in\left[0.95 \times 10^{-4} F \sim 1.05 \times 10^{-4} F\right], R_{1} \in\left[0.45 \times 10^{3} \Omega \sim 0.55 \times 10^{3} \Omega\right]$ \\
\hline
\end{tabular}

The amplitude distributions of the five fault types of the Sallen Key filter are shown in Figure 8.

The 100 sets of sample data are used as training samples, and the remaining 100 sets are used as test samples. The fault diagnosis of the Sallen Key filter is carried out by the LSSVM fusion algorithm. The order of the polynomial function is 3, and the width of the Gaussian radial basis function is $2 \times 10^{-3}$. In addition, the single LSSVM and multikernel LSSVM [39] multiclassification algorithm are used for comparative experiments. 


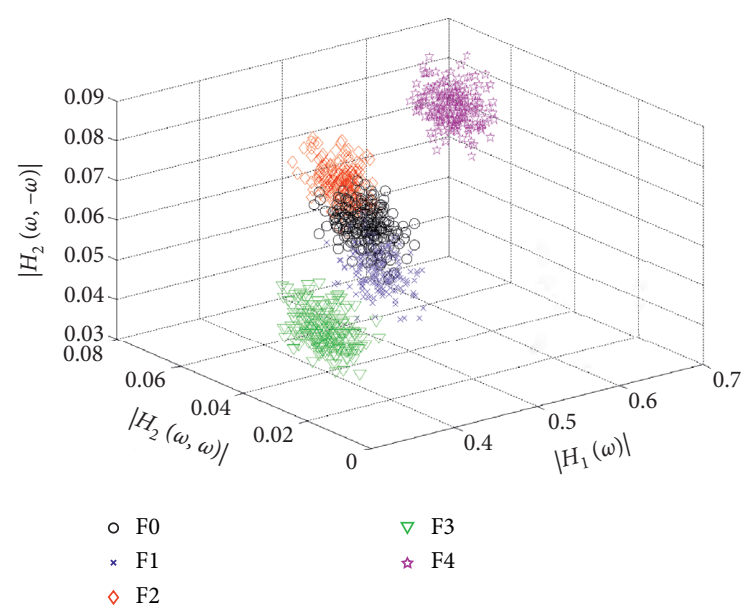

Figure 5: The amplitude distributions of the five fault types.

TABLE 3: The fault recognition rate of RC circuit.

\begin{tabular}{lcccc}
\hline $\begin{array}{l}\text { Fault } \\
\text { number }\end{array}$ & Fault type & $\begin{array}{c}\text { LSSVM fusion } \\
\text { algorithm }\end{array}$ & $\begin{array}{c}\text { Recognition rate } \\
\text { Single } \begin{array}{c}\text { LSM (polynomial kernel } \\
\text { function) }\end{array}\end{array}$ & $\begin{array}{c}\text { Single LSSVM (Gaussian radial basis kernel } \\
\text { function) }\end{array}$ \\
\hline F0 & Normal & 93 & 91 & 86 \\
F1 & C increased & 99 & 98 & 95 \\
F2 & C decreased & 90 & 90 & 88 \\
F3 & $R_{1}$ increased & 100 & 100 & 96 \\
F4 & $\mathrm{R}_{1}$ decreased & 100 & 100 & 100 \\
\hline
\end{tabular}

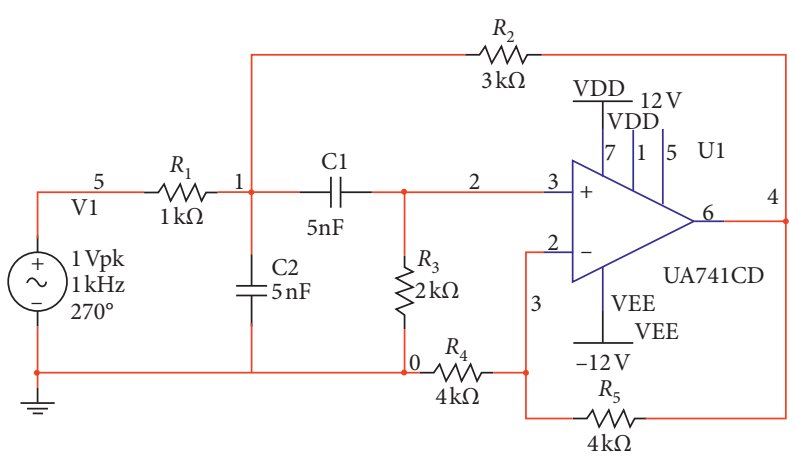

Figure 6: A Sallen Key filter circuit.

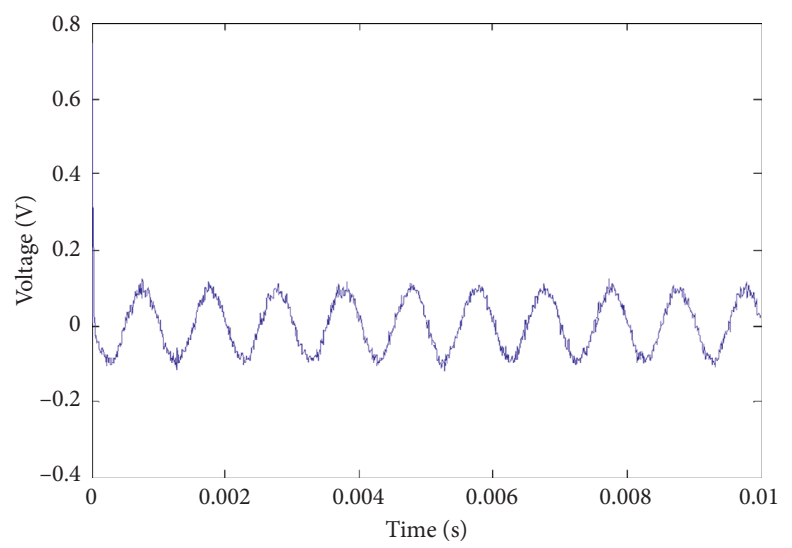

Figure 7: The output of Sallen Key filter. 


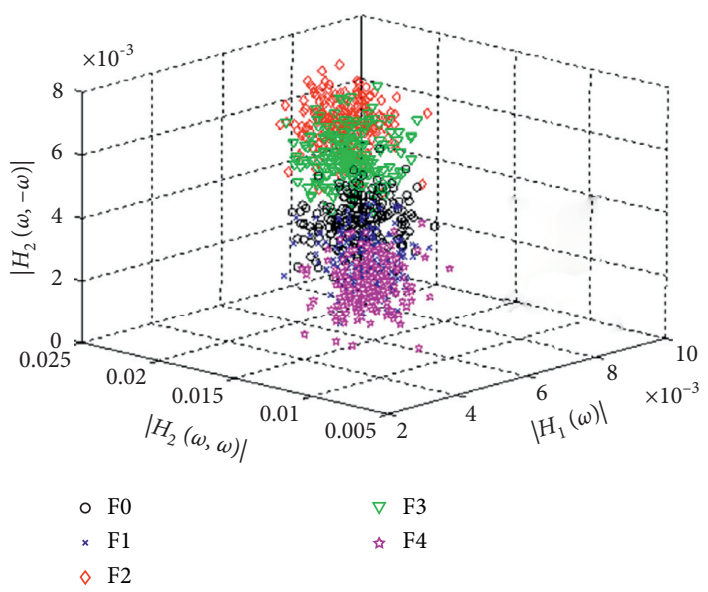

Figure 8: The amplitude distributions of the five fault types of Sallen Key filter.

TABLE 4: The fault recognition rate of Sallen Key filter.

\begin{tabular}{|c|c|c|c|c|c|}
\hline \multirow[b]{2}{*}{$\begin{array}{l}\text { Fault } \\
\text { number }\end{array}$} & \multirow[b]{2}{*}{ Fault type } & \multicolumn{4}{|c|}{ Recognition rate } \\
\hline & & $\begin{array}{l}\text { LSSVM fusion } \\
\text { algorithm (\%) }\end{array}$ & $\begin{array}{l}\text { Single LSSVM (polynomial } \\
\text { kernel function) }(\%)\end{array}$ & $\begin{array}{l}\text { Single LSSVM (Gaussian radial basis } \\
\text { kernel function) (\%) }\end{array}$ & $\begin{array}{l}\text { Multikernel } \\
\text { LSSVM (\%) }\end{array}$ \\
\hline F0 & Normal & 94 & 85 & 88 & 90 \\
\hline F1 & $\begin{array}{c}R_{4} \\
\text { increased }\end{array}$ & 81 & 69 & 71 & 73 \\
\hline $\mathrm{F} 2$ & $\begin{array}{c}R_{4} \\
\text { decreased }\end{array}$ & 96 & 87 & 89 & 88 \\
\hline F3 & $\begin{array}{c}R_{5} \\
\text { increased }\end{array}$ & 97 & 86 & 88 & 91 \\
\hline $\mathrm{F} 4$ & $\begin{array}{c}\mathrm{R}_{5} \\
\text { decreased }\end{array}$ & 83 & 72 & 75 & 76 \\
\hline
\end{tabular}

The fault recognition rate is shown in Table 4. The recognition rates of the LSSVM fusion algorithm are significantly higher than those of the single LSSVM and multikernel LSSVM classifiers.

Therefore, the proposed diagnosis method for nonlinear analog circuit based on the generalized frequency response function and LSSVM fusion algorithm has good diagnostic performance.

\section{Conclusions}

According to generalized frequency response function and LSSVM classifier fusion, a fault diagnosis method for nonlinear analog circuits is presented in this paper. The sinusoidal signal is used as input, and the first two-order generalized frequency response functions of the circuit system are directly estimated by the time-domain measurements. The amplitudes of the generalized frequency response functions are used as the feature parameters. In order to improve the accuracy of diagnosis, a classifier fusion algorithm based on LSSVM is presented for fault identification. The polynomial kernel function and Gaussian radial basis function are used to construct two different LSSVM classifiers, and then the decision fusion is carried out by the binary LSSVMs of these two classifiers. Two fault diagnosis experiments for RC circuit and Sallen Key filter are used to verify the effectiveness of the proposed method. The results show that the method can achieve good fault diagnosis performance.

For fault diagnosis of complex circuits in real time applications, the diagnosis can be carried out by the proposed method only when the input signal is sinusoidal. Furthermore, the fault diagnosis of analog circuit under the excitation of multitone signal will be studied.

\section{Data Availability}

The data used to support the findings of this study are available from the corresponding author upon request.

\section{Conflicts of Interest}

The authors declare that there are no conflicts of interest regarding the publication of this paper.

\section{Acknowledgments}

This research was funded by the Natural Science Basic Research Project of Shaanxi, grant no. 2019JM-339. 


\section{References}

[1] M. Tadeusiewicz and S. Halgas, "A method for local parametric fault diagnosis of a broad class of analog integrated circuits," IEEE Transactions on Instrumentation and Measurement, vol. 67, no. 2, pp. 328-337, 2018.

[2] X. Yuan, Z. Liu, Z. Miao, Z. Zhao, F. Zhou, and Y. Song, "Fault diagnosis of analog circuits based on IH-PSO optimized support vector machine," IEEE Access, vol. 7, pp. 137945137958, 2019.

[3] Z. Liu, Z. Jia, C.-M. Vong, S. Bu, J. Han, and X. Tang, "Capturing high-discriminative fault features for electronicsrich analog system via deep learning," IEEE Transactions on Industrial Informatics, vol. 13, no. 3, pp. 1213-1226, 2017.

[4] X. S. Gan, W. M. Gao, Z. Dai, and W. D. Liu, "Research on WNN soft fault diagnosis for analog circuit based on adaptive UKF algorithm," Applied Soft Computing, vol. 50, pp. 252259, 2017.

[5] A. Kumar and A. P. Singh, "Fuzzy classifier for fault diagnosis in analog electronic circuits," ISA Transactions, vol. 52, no. 6, pp. 816-824, 2013.

[6] L. F. Yuan, Y. G. He, J. Y. Huang, and Y. C. Sun, "A new neural-network-based fault diagnosis approach for analog circuits by using kurtosis and entropy as a preprocessor," IEEE Transactions on Instrumentation and Measurement, vol. 59, no. 3, pp. 586-595, 2010.

[7] M. Aminian and F. Aminian, "A modular fault-diagnostic system for analog electronic circuits using neural networks with wavelet transform as a preprocessor," IEEE Transactions on Instrumentation and Measurement, vol. 56, no. 5, pp. 1546-1554, 2007.

[8] Y. Xiao and L. Feng, "A novel linear ridgelet network approach for analog fault diagnosis using wavelet-based fractal analysis and kernel PCA as preprocessors," Measurement, vol. 45, no. 3, pp. 297-310, 2012.

[9] K. Worden, W. E. Becker, T. J. Rogers, and E. J. Cross, "On the confidence bounds of Gaussian process NARX models and their higher-order frequency response functions," Mechanical Systems and Signal Processing, vol. 104, pp. 188-223, 2018.

[10] Z. A. Khan, E. Zenteno, P. Händel, and M. Isaksson, "Extraction of the third-order $3 \times 3$ MIMO Volterra kernel outputs using multitone signals," IEEE Transactions on Microwave Theory and Techniques, vol. 66, no. 11, pp. 4985-4999, 2018.

[11] S. A. Billings and K. M. Tsang, "Spectral analysis for nonlinear systems, part I: parametric non-linear spectral analysis," Mechanical Systems and Signal Processing, vol. 3, no. 4, pp. 319-339, 1989.

[12] K. Worden, G. Manson, and G. R. Tomlinson, "A harmonic probing algorithm for the multi-input Volterra series," Journal of Sound and Vibration, vol. 201, no. 1, pp. 67-84, 1997.

[13] C. H. Tseng, "A mixed-domain method for identification of quadratically nonlinear systems," IEEE Transactions on Signal Processing, vol. 45, no. 4, pp. 1013-1024, 1997.

[14] F. Kuech and W. Kellermann, "Partitioned block frequencydomain adaptive second-order Volterra filter," IEEE Transactions on Signal Processing, vol. 53, no. 2, pp. 564-575, 2005.

[15] K. I. Kim and E. J. Powers, "A digital method of modeling quadratically nonlinear systems with a general random input," IEEE Transactions on Acoustics, Speech, and Signal Processing, vol. 36, no. 11, pp. 1758-1769, 1988.

[16] M. Alizadeh, S. Amin, and D. Rönnow, "Measurement and analysis of frequency-domain Volterra kernels of nonlinear dynamic \$3 \times 3\$ MIMO systems," IEEE Transactions on
Instrumentation and Measurement, vol. 66, no. 7, pp. 18931905, 2017.

[17] B. Zhang and S. A. Billings, "Volterra series truncation and kernel estimation of nonlinear systems in the frequency domain," Mechanical Systems and Signal Processing, vol. 84, pp. 39-57, 2017.

[18] R. M. Lin and T. Y. Ng, "A new method for the accurate measurement of higher-order frequency response functions of nonlinear structural systems," ISA Transactions, vol. 81, pp. 270-285, 2018.

[19] R. M. Lin and T.-Y. Ng, "Identification of Volterra kernels for improved predictions of nonlinear aeroelastic vibration responses and flutter," Engineering Structures, vol. 171, pp. 15-28, 2018.

[20] J. Zhang and J. Cao, "Estimation of multi-order spectra for nonlinear closed-loop systems," Asian Journal of Control, vol. 20, no. 1, pp. 286-297, 2018.

[21] L. M. Li and S. A. Billings, "Estimation of generalized frequency response functions for quadratically and cubically nonlinear systems," Journal of Sound and Vibration, vol. 330, no. 3, pp. 461-470, 2011.

[22] J. L. Zhang and J. F. Cao, "Nonlinear circuit fault diagnosis based on simplified estimation of generalized frequency response function," in Proceedings of the 32nd Chinese Control And Decision Conference, pp. 698-702, Hefei, China, 2020.

[23] B. Marcin, "Ensembles of instance selection methods: a comparative study," International Journal of Applied Mathematics and Computer Science, vol. 29, no. 1, pp. 151-168, 2019.

[24] C. Pawel, "A case study in text mining of discussion forum posts: classification with bag of words and global vectors," International Journal of Applied Mathematics and Computer Science, vol. 28, no. 4, pp. 787-801, 2018.

[25] H. Cui, L. Zhang, R. Kang, and X. Lan, "Research on fault diagnosis for reciprocating compressor valve using information entropy and SVM method," Journal of Loss Prevention in the Process Industries, vol. 22, no. 6, pp. 864-867, 2009.

[26] K.-Y. Chen, L.-S. Chen, M.-C. Chen, and C.-L. Lee, "Using SVM based method for equipment fault detection in a thermal power plant," Computers in Industry, vol. 62, no. 1, pp. 42-50, 2011.

[27] S. Ekici, "Support vector machines for classification and locating faults on transmission lines," Applied Soft Computing, vol. 12, no. 6, pp. 1650-1658, 2012.

[28] U. B. Parikh, B. Biswarup Das, and R. P. Prakash Maheshwari, "Combined wavelet-SVM technique for fault zone detection in a series compensated transmission line," IEEE Transactions on Power Delivery, vol. 23, no. 4, pp. 1789-1794, 2008.

[29] J. Ni, C. Zhang, and S. X. Yang, "An adaptive approach based on KPCA and SVM for real-time fault diagnosis of HVCBs," IEEE Transactions on Power Delivery, vol. 26, no. 3, pp. 1960-1971, 2011.

[30] J. Huang, X. G. Hu, and F. Yang, "Support vector machine with genetic algorithm for machinery fault diagnosis of high voltage circuit breaker," Measurement, vol. 44, no. 6, pp. 1018-1027.

[31] P. Konar and P. Chattopadhyay, "Bearing fault detection of induction motor using wavelet and support vector machines (SVMs)," Applied Soft Computing, vol. 11, no. 6, pp. 4203-4211, 2011.

[32] S. S. Ben, K. Bacha, and A. Chaari, "Support vector machine based decision for mechanical fault condition monitoring in induction motor using an advanced Hilbert-Park transform," ISA Transactions, vol. 51, no. 5, pp. 566-572, 2012. 
[33] P. B. Tribeni and D. Swagatam, "Multi-sensor data fusion using support vector machine for motor fault detection," Information Sciences, vol. 217, pp. 96-107, 2012.

[34] R. Jegadeeshwaran and V. Sugumaran, "Fault diagnosis of automobile hydraulic brake system using statistical features and support vector machines," Mechanical Systems and Signal Processing, vol. 52-53, pp. 436-446, 2015.

[35] F. N. Rudsari, A. A. Razi-Kazemi, and M. A. Shoorehdeli, "Fault analysis of high-voltage circuit breakers based on coil current and contact travel waveforms through modified SVM classifier," IEEE Transactions on Power Delivery, vol. 34, no. 4, pp. 1608-1618, 2019.

[36] S. T. Kandukuri, J. S. L. Senanyaka, V. K. Huynh, and K. G. Robbersmyr, "A two-stage fault detection and classification scheme for electrical pitch drives in offshore wind farms using support vector machine," IEEE Transactions on Industry Applications, vol. 55, no. 5, pp. 5109-5118, 2019.

[37] J. A. K. Suykens and J. Vandewalle, "Least square support vector machine classifiers," Neural Processing Letters, vol. 9, no. 3, pp. 293-300, 1999.

[38] S. A. Billings and J. C. Peyton Jones, "Mapping non-linear integro-differential equations into the frequency domain," International Journal of Control, vol. 52, no. 4, pp. 863-879, 1990.

[39] V. Ceperic, G. Gielen, and A. Baric, "Sparse multikernel support vector regression machines trained by active learning," Expert Systems with Applications, vol. 39, no. 12, pp. 11029-11035, 2012. 